\title{
Focal adhesion kinase regulates the phosphorylation protein tyrosine phosphatase- $\alpha$ at Tyr789 in breast cancer cells
}

\author{
XU-QIAN FANG ${ }^{1}$, XIANG-FAN LIU ${ }^{2}$, LING YAO ${ }^{3}$, CHANG-QIANG CHEN $^{1}$, JIA-FEI LIN ${ }^{1}$, \\ ZHI-DONG GU ${ }^{1}$, PEI-HUA NI ${ }^{2}$, XIN-MIN ZHENG ${ }^{3,4}$ and QI-SHI FAN ${ }^{1}$ \\ ${ }^{1}$ Department of Clinical Laboratory, Ruijin North Hospital, Ruijin Hospital; ${ }^{2}$ Faculty of Medical Laboratory Science; \\ ${ }^{3}$ Department of Biochemistry and Molecular Biology, Shanghai JiaoTong University School of Medicine, \\ Shanghai 200025, P.R. China; ${ }^{4}$ Department of Molecular Biology and \\ Genetics, Cornell University, Ithaca, NY 14853-2703, USA
}

Received June 3, 2014; Accepted November 7, 2014

DOI: $10.3892 / \mathrm{mmr} .2015 .3262$

\begin{abstract}
Protein tyrosine phosphatase (PTP)- $\alpha$ regulates the phosphorylation of focal adhesion kinase (FAK), which is important in cellular signal transduction and integration of proteins. It has been demonstrated that a FAK-Del33 mutation (deletion of exon 33; KF437463) in breast cancer tissues regulates cell migration through FAK/Src signaling activation. However, the detailed pathway for Src activation with FAK-Del33 remains to be elucidated. The present study used a retroviral expression system to examine changes in PTP $\alpha$ phosphorylation affected by the FAK-Del33 protein in breast cancer cells. Small interfering (si)RNA targeting PTP $\alpha$ interfered with the phosphorylation of Src. Wound-healing and migration assays were performed to identify cell morphology and quantitative analysis was performed by examining band color depth in western blot analysis. Significant differences were observed in the phosphorylation level of PTP $\alpha$ at Tyr789 between the FAK-Del33 and the wild-type breast cancer cells, suggesting that FAK regulated the phosphorylation level of PTP $\alpha$ at Tyr789 in breast cancer mutant FAK-Del33 cells. The gene expression profile with FAK siRNA did not alter the levels of phosphorylation in other mutants, including autophosphorylation disability (Y397F), ATP kinase dominant negative (K454R) and protein 4.1, ezrin, radixin, moesin domain attenuate $(\Delta 375)$. FAK RNAi inhibited the activity of the FAK-Del33 at the Src site and rescued the
\end{abstract}

Correspondence to: Professor Qi-Shi Fan, Department of Clinical Laboratory, Ruijin North Hospital, Ruijin Hospital, Shanghai JiaoTong University School of Medicine, 197 Ruijin Second Road, Shanghai 200025, P.R. China

E-mail: qishifan@126.com

Professor Xin-Min Zheng, Department of Molecular Biology and Genetics, 265 Biotechnology Building, Cornell University, 410 Thurston Avenue, Ithaca, NY 14853-2703, USA

E-mail: xmz27@cornell.edu

Key words: focal adhesion kinase with exon 33 deletion, protein tyrosine phosphatase- $\alpha$, regulates phosphorylation elevated cell migration and invasion. The present study demonstrated for the first time, to the best of our knowledge, an increase in the phosphorylation level of PTP $\alpha$-Tyr789 by its upstream activator, FAK-Del33, leading to Src activation in certain breast cancer cells, which has significant implications for metastatic potential.

\section{Introduction}

Protein tyrosine kinases (PTK) and protein tyrosine phosphatases (PTP) regulate tyrosine phosphorylation, which is an important cellular mechanism that regulates intracellular signaling pathways to control a number of cellular activities, including proliferation, growth and differentiation (1). Focal adhesion kinase (FAK), a non-receptor binding to tyrosine kinase, functions as a scaffold and coordinating center, which may regulate focal adhesion formation and disassembly. This has been demonstrated in a previous study, which investigated actin fiber stress and cytoskeletal organization (2). The most important site for the regulation of FAK phosphorylation is Tyr397. This mediates one key intramolecular regulatory interaction between the protein 4.1, ezrin, radixin, moesin (FERM) and kinase domains of FAK $(3,4)$. In the integrin signaling pathway, FAK-Tyr397, accompanied by auto-phosphorylation reconstructs the Src family kinases (SFK)/FAK composite, which leads to further phosphorylation and maximal activation of FAK (2).

The important role of SFK in activating FAK has been demonstrated by the decreasing levels of FAK auto-phosphorylation following knock out of the Src, Fyn and Yes gene of SFKs in fibroblasts or by shielding the PTP gene in response to integrin engagement $(5,6)$. In terms of the molecular mechanisms, evidence suggests that the FERM domain is responsible for FAK auto-inhibition by interacting with the kinase domain. The FERM domain was observed to switch from binding to the kinase domain to binding with heterologous protein or lipid partners, which led to FAK-Tyr397 auto-phosphorylation $(3,6,7)$.

PTP $\alpha$ may also regulate the levels of FAK phosphorylation and a previous study suggested that $\mathrm{PTP} \alpha$ and other PTP family members may catalyze the dephosphorylation 
of Src-Tyr527 by its own activity (8-10). During the mitotic process, phosphorylated Src-Tyr527 is able to activate the expression of Src, the regulation of which is mediated by $\operatorname{PTP} \alpha$ (11). However, it has also been suggested that PTP $\alpha$-mediated Src activation during mitosis was dependent on PP2A-mediated dephosphorylation of Ser204 in the juxtamembrane region of PTP $\alpha$ rather than Tyr789 (12). Therefore, the role of Src-Try789 phosphorylated by PTP $\alpha$ requires further investigation.

In our previous study, a novel splicing mutant, FAK-Del33, was identified in breast and thyroid cancer by sequencing (13). The mutation frequency of The FAK-De133 gene was found to be $14.2 \%(3 / 21)$ in breast carcinoma; in addition, when this gene was introduced into normal breast cell lines, it was reported to promote cell migration and invasion (13). This may be caused by FAK-Tyr397 hyperphosphorylation and enhancement of FAK-Src in downstream signaling pathways.

The present study investigated changes in the levels of amino acid phosphorylation in the signaling pathway of breast cancer cells and the activity of Src by western blot analysis. A novel pathway, in which the FAK-Del33 protein may activate c-Src through PTP $\alpha$ phosphorylation at the Tyr789 site, was identified. This was the first study, to the best of our knowledge, to demonstrate the effect of PTP $\alpha$ phosphorylation in a FAK mutant.

\section{Materials and methods}

Cell lines. The MDA-MB-468 and MDA-MB-435s cell lines were isolated from a breast cancer patient and melanoma derived cells (MDA-MB-435s) were obtained from American Type Culture Collection (Manassas, VA, USA) and cultured according to the manufacturer's instructions. The HEK293T cell line was also obtained from American Type Culture Collection. The FAK-Del33 mutant cells were obtained from breast cancer mutation cells lacking exon 33 for FAK and were stored at the Department of Clinical Laboratory of Shanghai Ruijin Hospital (Shanghai, China).

Construction and identification of the expression system. The FAK-Del33 mutant cell suspension was harvested after $48 \mathrm{~h}$ in culture and the nucleic acids were then extracted using phenol-chloroform extraction technology. In brief, $500 \mu 1$ phenol:chloroform:isoamyl alcohol (25:24:1) was added to the cell suspension and it was vortexed for $20 \mathrm{sec}$, then centrifuged at $16,000 \mathrm{x} \mathrm{g}$ for $5 \mathrm{~min}$ at room temperature. The supernatant was transferred to a fresh tube and ethanol precipitation was performed by adding cold $95 \%$ ethanol, incubating for $1 \mathrm{~h}$ at $-20^{\circ} \mathrm{C}$, centrifuging at $16,000 \mathrm{x}$ g for $15 \mathrm{~min}$, decanting the supernatant, adding $80 \%$ ethanol and repeating the centrifugation. Polymerase chain reaction (PCR) was performed to amplify the FAK-Del33 gene, using the following primers: forward 5'-TCA GAA ACA GAT GAT TTT GCT GAG AT T ATA GAT GA-3' and reverse 5'-AGC AAA ATC ATC T GT TTC TGA CAC AGA GA-3'. Furthermore, BamHI and Not I restriction sites were introduced at the $5^{\prime}$ and $3^{\prime}$ ends, respectively. The purified PCR fragment was inserted into multiple cloning sites in pCDNA3.1 (+). Construction of the expression plasmids used in the present study are shown in Fig. 1A.
Retroviral expression system. The pGIPZ lentiviral vector-based expression system (Open Biosystems, Inc., Victoria, Australia) was used to clone and express the FAT-wild-type (Wt) and FAK-del33 (exon 33 of FAK deletion) genes in the cells, according to the manufacturers instructions, which packaged the lentivirus and infected the HEK293T cells to culture and amplify the virus.

Wound-healing assay. A wound-healing assay was performed to detect changes in the motility and migration of the MDA-MB-468 and MDA-MB-435s cell lines. The FAK-Del33 mutant was used as a positive control. Cells, which were infected with the recombinant virus and empty vector were used as a negative control.

The cells were seeded $\left(3 \times 10^{5}\right)$ into $60 \mathrm{~mm}^{2}$ plates and cultured for $12 \mathrm{~h}$ at $37^{\circ} \mathrm{C}$. A vertical scratch was made using a p20 pipette tip in the middle of the plate to remove cells from this area. Following this, the cells were washed three times using phosphate-buffered saline (PBS) and cultured in serum-free medium. Images of the wound-healing distance were captured for each cell line at $0,6,12$ and $24 \mathrm{~h}$ using an inverted microscope (IX73; Olympus Corp., Tokyo, Japan).

Migration assay. The cells $\left(3 \times 10^{5}\right)$ were cultured in $60 \mathrm{~mm}^{2}$ plates containing Transwell filters with an $8 \mu \mathrm{m}$ pore size (Corning, Inc., New York, NY, USA) coated with $10 \mu \mathrm{g} / \mathrm{ml}$ fibronectin for $12 \mathrm{~h}$. The culture medium (Dulbecco's modified Eagle's medium) was designed with a serum-free upper layer containing $0.1 \%$ bovine serum albumin (BSA) and a lower layer containing 20\% fetal bovine serum (Gibco-BRL, Gaithersburg, MD, USA) in different chambers. After $24 \mathrm{~h}$, the bottom of the filter was stained with Giemsa (Sangon Biotech Co., Ltd, Shanghai, China) to calculate the efficiency of the cell migration.

Sodium dodecyl sulfate polyacrylamide gel electrophoresis (SDS-PAGE) and western blot analysis. The FAK-Del33 mutant and FAK-Wt cell suspensions were harvested and centrifuged at $1,000 \mathrm{x} g$ for $10 \mathrm{~min}$ (Thermo Fisher Scientific, Waltham, MA, USA). The supernatant was discarded and the pellet was resuspended in lysis buffer [20 mM Tris ( $\mathrm{pH} 7.5$ ), $150 \mathrm{mM} \mathrm{NaCl}, 1 \%$ Triton X-100; Beyotime Institiute of Technology, Jiangsu, China], containing $1 \%$ protease inhibitor cocktail (Sigma-Aldrich, St Louis, MO, USA), $25 \mathrm{mM} \mathrm{NaF} \mathrm{(Sigma-Aldrich)} \mathrm{and}$ $1 \mathrm{mM} \mathrm{Na}_{3} \mathrm{VO}_{4}$ (Sigma-Aldrich).

The mixture was freeze-thawed four times at $-80^{\circ} \mathrm{C}$ prior to centrifuging at $10,000 \mathrm{x}$ g for $30 \mathrm{~min}$ at $4^{\circ} \mathrm{C}$ (Thermo Fisher Scientific). The supernatant containing FAK-Del33 protein was collected and analyzed using 8\% SDS-PAGE. The FAK-Wt breast cancer cells were used for comparison. Following SDS-PAGE, the proteins were transferred onto a polyvinylidene difluoride membrane (Millipore, Billerica, MA, USA).

The membrane was blocked using 5\% BSA in Tris- $\mathrm{HCl}$ buffered saline containing $0.05 \%$ Tween- 20 for $2 \mathrm{~h}$ at $25^{\circ} \mathrm{C}$. The membrane was then incubated with the following primary monoclonal antibodies at $4^{\circ} \mathrm{C}$ overnight $(1: 1,000)$ : Mouse anti-PTP $\alpha$, rabbit anti-phospho-PTP $\alpha$ (Tyr789), rabbit anti-Src, rabbit anti-phospho-Src (Tyr527); rabbit anti-non-phospho-Src (Tyr527); rabbit anti-phospho-Src (tyr416) and rabbit anti-PTP1B, followed by incubation at room temperature for $2 \mathrm{~h}$ with anti-rabbit 
A

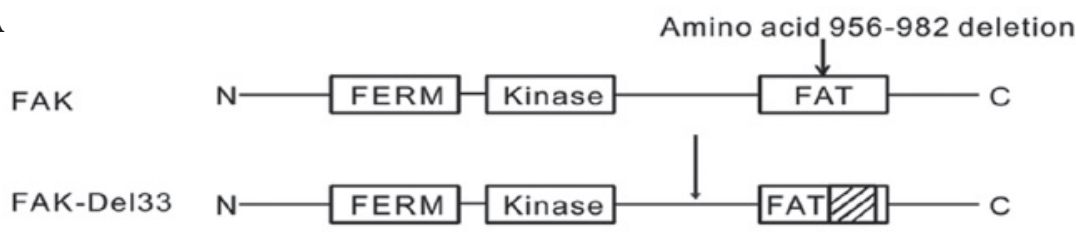

B

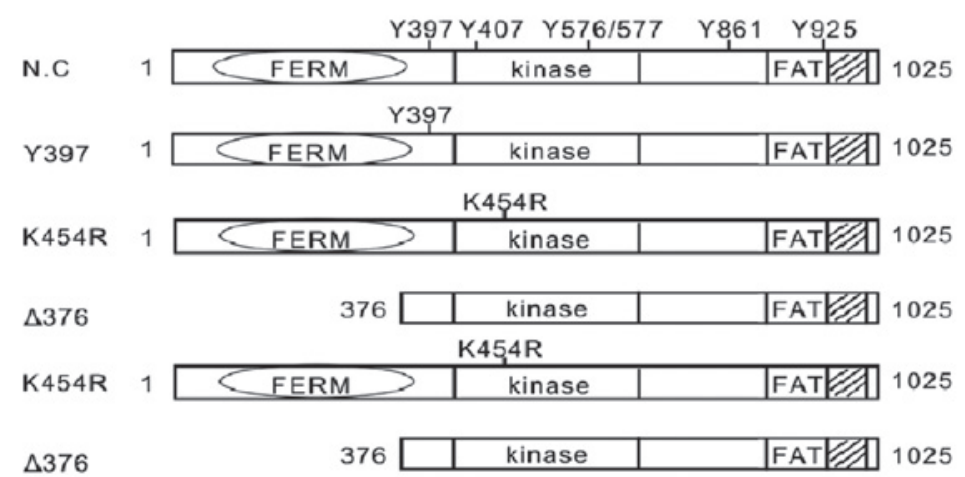

C

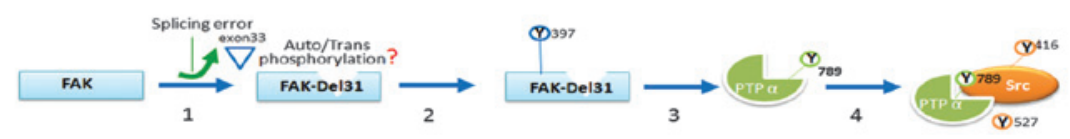

Figure 1. Schematic diagram of FAK and FAK-Del33 (A) FAK-Del33 mutation involved deletion of exon33 of FAT. (B) PTP $\alpha$ analysis revealed five mutation patterns of FAK compared with FAK-Del33. (C) Model of c-Src activation in cells with FAK-Del33. FAK, focal adhesion kinase; FAK-Del33, FAK with exon 33 deletion; FAT, focal adhesion kinase targeting domain; PTP $\alpha$, protein tyrosine phosphatase- $\alpha$; FERM, protein 4.1, ezrin, radixin, moesin.

or anti-mouse immunoglobulin G secondary antibodies. All antibodies were purchased from Cell Signaling Technology, Inc. (Beverly, MA, USA). GADPH levels were used as an internal standard. The protein bands were detected using enhanced chemiluminescence (Bestbio, Shanghai, China). The Wt breast cancer cells were used as a negative control.

siRNA-induced apoptosis. The following siRNA sequences were used to target: PTP $\alpha$ forward 5'-GCU GGG AGC CAU UCC AAU UdTdT-3' and reverse primers PTP $\alpha$ 5'GCU AGG AGC UAU UCC GAU U dTdT-3', PTP1B 5'-AAU ACA GUG CGA CAG CUA GAA dTdT-3' and control siRNA 5'-AAU UAA CCU UAC CGA GGG UCG dTdT-3' (14). All the siRNA duplexes were synthesized by GenePharma, Inc. (Shanghai, China) using 2'-ACE protection chemistry. The Stemfect ${ }^{\mathrm{TM}}$ RNA Transfection kit (Stemgent, Cambridge, MA, USA) was used to transfect the siRNA into the cells. Fig. 1B shows the amino acid positions in the FAK protein.

Statistical analysis. Statistical analysis was performed using SPSS 17.0 software (SPSS, Inc., Chicago, IL, USA). Differences between the two groups were evaluated using a one-way analysis of variance. A $\chi^{2}$ test was used to calculate the significance of detection rates between the two groups. $\mathrm{P}<0.05$ was considered to indicate a statistically significant difference.

\section{Results}

Wound-healing and migration assay. The healing rate of the FAK-Del33 mutant was faster than that of MDA-MB-468 and MDA-MB-435s cells. Following infection with a lentivirus contaning the FAK-Del33 gene, the healing efficiency of the MDA-MB-468 and MDA-MB-435s cells was higher compared with those observed in the cells containing the empty vector. The FAK-Del33 protein may regulate cell growth. The results obtained from the migration assays supported the hypothesis that this protein also regulates cell motility and migration (Fig. 2).

Western blot analysis. The main autophosphorylation of FAK, Src-Tyr397, was overexpressed in the FAK-Del33 mutant compared with the FAK-Wt cells. The results revealed $\sim 60-80 \%$ of the Src-Tyr530 was dephosphorylated in the MDA-MB-468 and MDA-MB-435s cells compared with the FAK-Del mutant cells. The phosphorylation levels of Src-Tyr419 were 1.90-3.07-fold higher in the FAK-Del33 mutant compared with the FAK-Wt cells (Fig. 3).

Significant differences were observed in the phosphorylation levels of PTP $\alpha$-Tyr789 between the FAK-Del33 mutant and the FAK-Wt cells (Fig. 4A) and an association between phosphorylated PTP $\alpha$-Tyr789 and dephosphorylated Src-Tyr530 was identified in the FAK-Del33 mutant cells (Fig. 4B).

To assess the interference of Grb2 in the phosphorylation of PTP $\alpha$-Tyr789, which was bound to the PTP $\alpha$ carboxyl terminus, western blot analysis was performed. The endogenous Grb2 antibody was obtained from lysates of the MDA-MB-468 cells by immunoprecipitation and its effects in the FAK-Del33 mutant cells were examined (Fig. 4C). The results demonstrated that PTP $\alpha$-Tyr789 exhibited the same level of phosphorylation and was not associated with the Grb2 antibody, suggesting that Grb2 is not involved in the dephosphorylation of Src-Tyr530. 
A

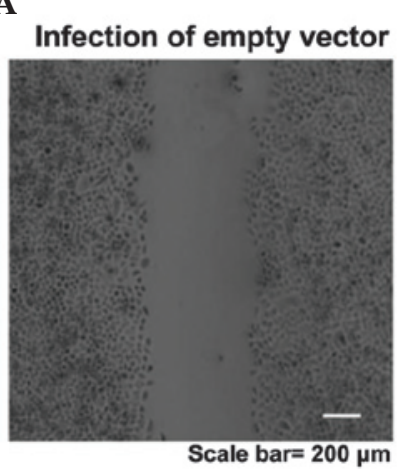

B

Infection of empty vector

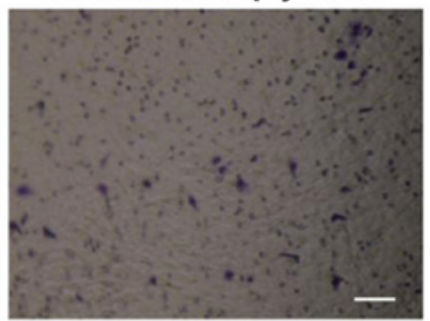

Scale bar= $100 \mu \mathrm{m}$
Infection of retroviral

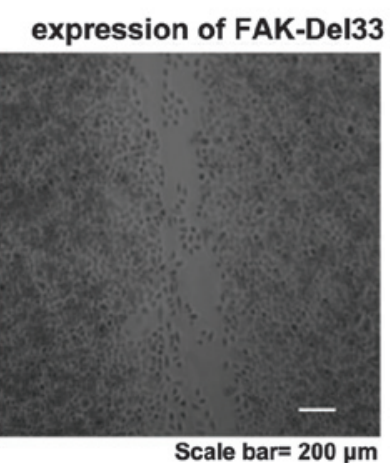

Infection of retroviral

expression of FAK-Del33

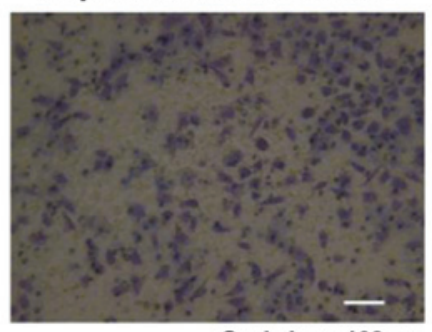

Scale bar= $100 \mu \mathrm{m}$
FAK-Del33 mutant as control

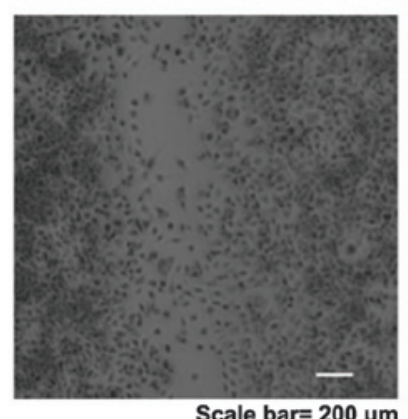

Scale bar= $200 \mu \mathrm{m}$

\section{FAK-Del33 mutant as control}

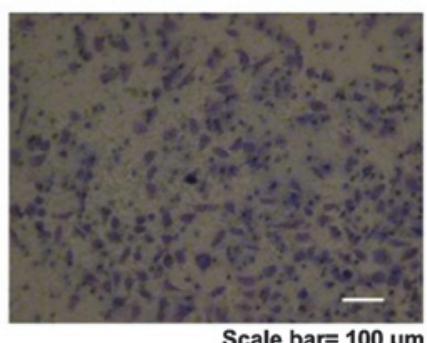

Figure 2. Analysis of images of cellular activity following infection with a lentivirus containing the FAK-Del33 gene. (A) Wound healing assay in the MDA-MB-468 cells. (B) Migration assay in the MDA-MB-435s cells. FAK, focal adhesion kinase; FAK-Del33; FAK with exon-33 deletion.

A

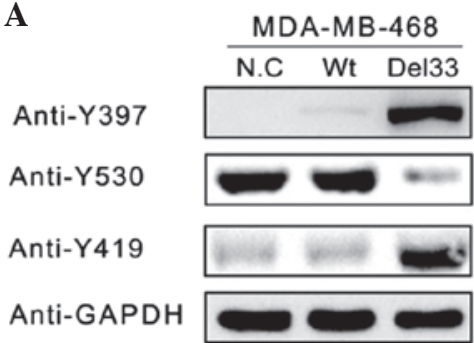

C

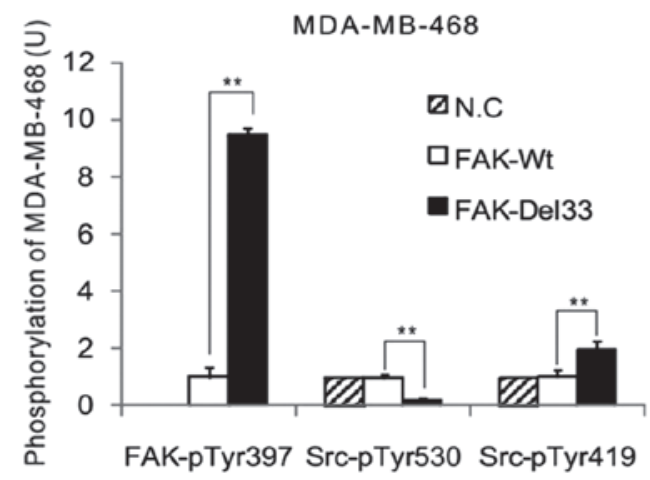

B

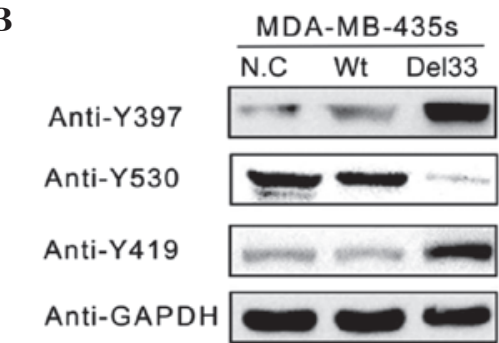

D

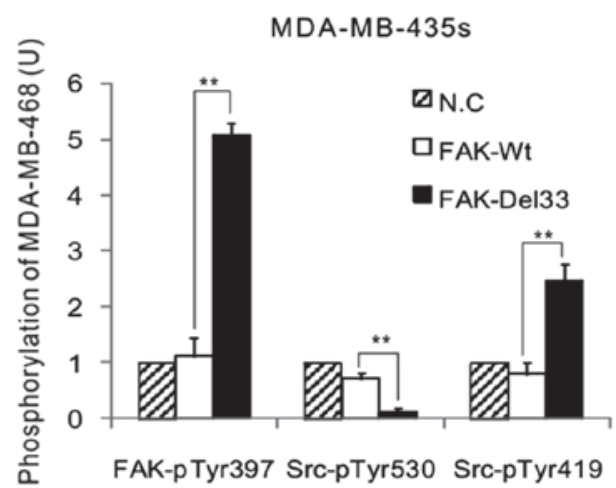

Figure 3. Changes in the phosphorylation levels of Src-pTyr530 and Src-Tyr419, between FAK-Wt and FAK-Del33 cells. (A) Western blot analysis of the MDA-MB-468 cells. (B) Western blot analysis of the MDA-MB-435s cells. (C) Quantification of the phosphorylation levels at specific protein sites in the MDA-MB-468 cells. (D) Quantification of phosphorylation levels at specific protein sites in the MDA-MB-435s cells. FAK, focal adhesion kinase; Wt, wild-type; FAK-Del33, FAK with exon-33 deletion; N.C, normal control.

Mutational analysis of amino acids. A total of three amino acid sites were selected for mutational analysis; Y397F (autophosphorylation site), K454R (ATP-binding site) and
$\Delta 375$ (deletion of FERM domain). As shown in Fig. 4D, all three FAK mutations in FAK-Del33 background failed to induce PTP $\alpha$-Tyr789 phosphorylation; this therefore 
A

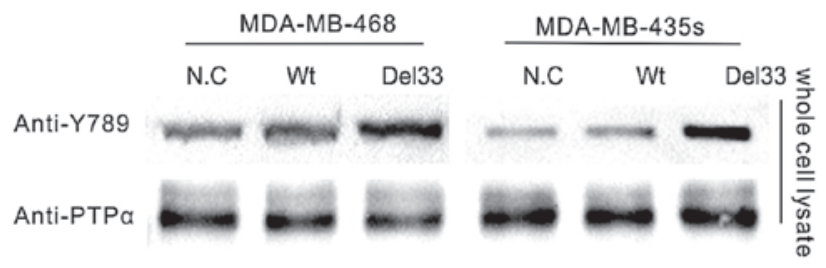

B
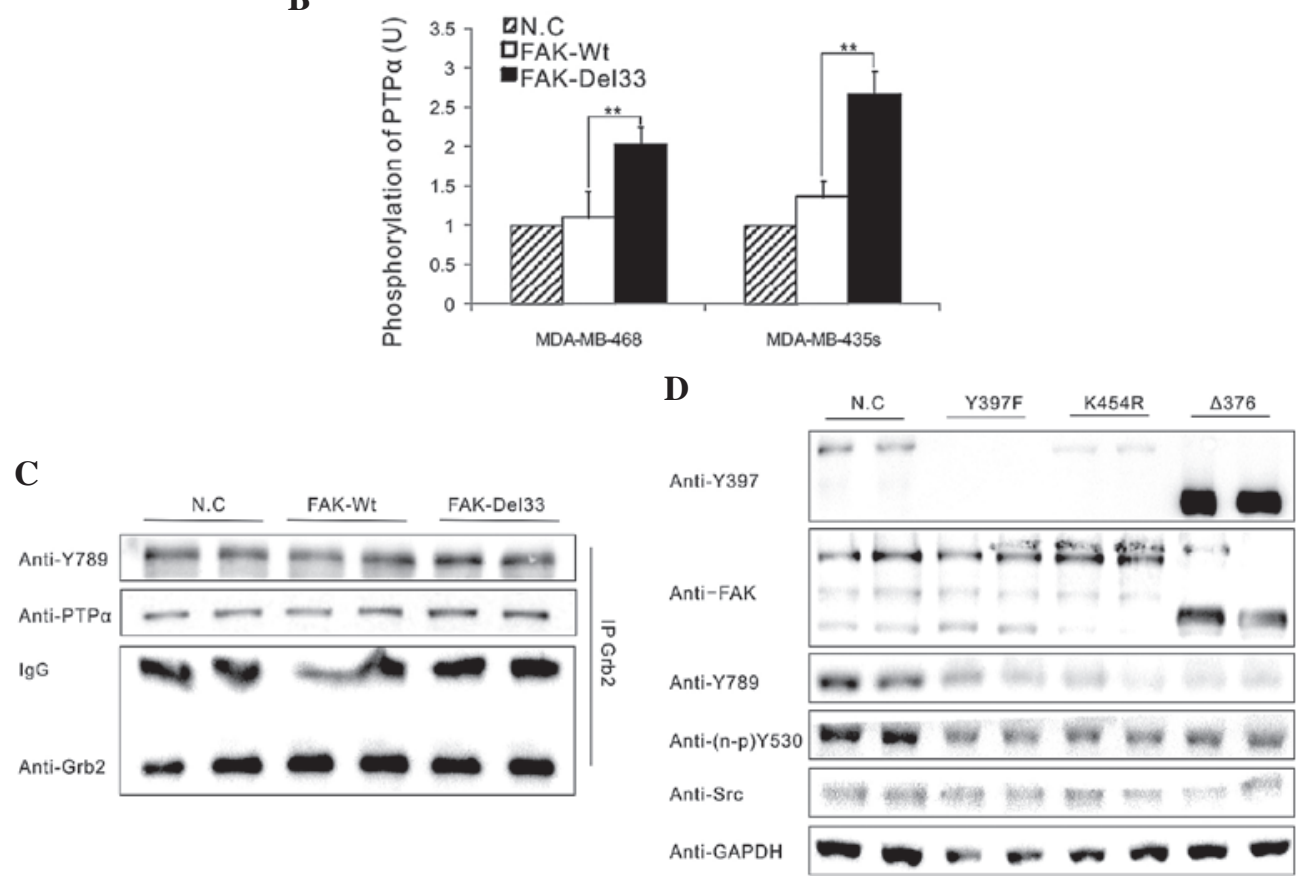

Figure 4. Association between the phosphorylation of Y789 and the activation of PTP $\alpha$ between FAK-Wt and FAK-Del33 cells. (A) Comparison of western blot analysis between the MDA-MB-468 and MDA-MB-435s cells. (B) Quantification of the levels of phosphorylation at specific protein sites in the MDA-MB-468 and MDA-MB-435s cells. (C) Western blot analysis of the effect of Grb2 on PTP $\alpha$ phosphorylation PTP $\alpha$. (D) Mutational analysis of amino acid sites following transfection of FAK-Del33, Y397F, K454R, $\Delta 375$ plasmids into MDA-MB-468 cells. PTP $\alpha$, protein tyrosine phosphatase- $\alpha$; FAK, focal adhesion kinase; FAK-Del33, FAK with exon 33 deletion; Wt, wild-type; N.C, normal control.

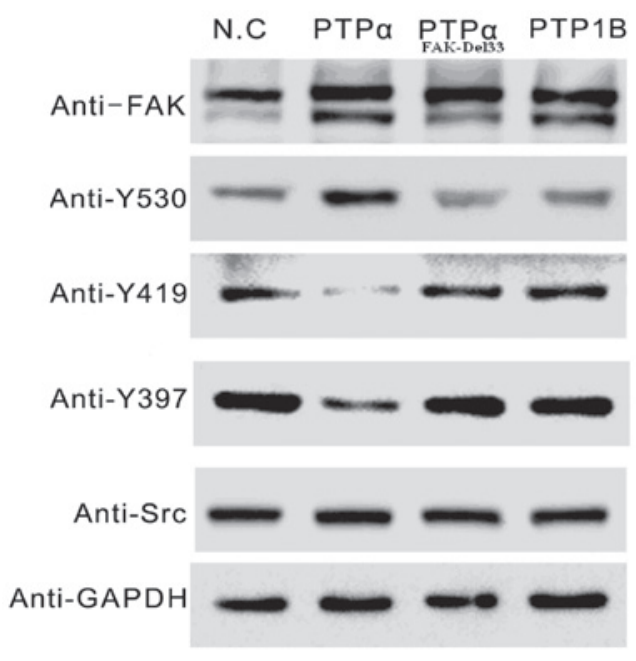

Figure 5. Western blot analysis of Src protein phosphorylation in response to siRNA interference at different sites. siRNA, small interfering RNA; N.C, negative control; PTP, protein tyrosine phosphatase; FAK, focal adhesion kinase; FAK-Del33, FAK with exon-33 deletion.

indicated that the ability of the FAK-Del33 protein to induce PTP $\alpha$-Tyr789 phosphorylation was dependent on Tyr397, K454R and the FERM domain.
RNAi in the FAK-Del33 mutant. The Src gene sites of Tyr530 dephosphorylation and Tyr419 phosphorylation changed significantly in the FAK-Del33 mutant. Following transfection of cells with PTP $\alpha$ siRNA, the expression efficiency of these two Src genes were reduced significantly. In addition, PTP $\alpha$ siRNA reduced the phosphorylation levels of Src-Tyr397 (Fig. 5), which was a feedback effect of the regulation of Src activation. However, these effects were not observed in the PTP1B siRNA. In addition, wound-healing and migration analyses revealed that the PTP $\alpha$ siRNA had an effect on the efficiency of cell migration and growth of the MDA-MB-468 cells Therefore, PTP $\alpha$ siRNA inhibited the FAK-Del33-mediated action on tumorigenesis.

A schematic diagram of a model of c-Src activation in cells with the FAK-Del33 mutation is shown in Fig. 1C, in which the deletion of exon 33 may be important in the regulation of Tyr789 phosphorylation. The identification of differences in protein binding sites and the exact role of the FAK mutant in PTP $\alpha$ regulation are important areas for future investigation.

\section{Discussion}

According to previous studies, PTP $\alpha$ acts as an upstream activator in the modulation of FAK phosphorylation $(13,14)$. The present study demonstrated a novel pathway, that the FAK-Del33 
mutation regulated PTP $\alpha$-Tyr789 phosphorylation, leading to Src-Tyr530 dephosphorylation. Previous studies involving PTP $\alpha$-deficient fibroblasts revealed that the phosphorylation of FAK, particularly at the Tyr397 auto-phosphorylation site can be identified in PTP $\alpha$-deficient fibroblasts and restored by reintroducing active, but not inactive, PTP $\alpha$. It was suggested that PTP $\alpha$ is required for maximal integrin-stimulated Src-PTK activity as an upstream regulator of FAK-Tyr397 phosphorylation $(15,16)$. The overexpression of PTP $\alpha$ has also been observed by increasing the tyrosine phosphorylation of FAK (17).

However, previous studies have demonstrated that FAK is required for PTP $\alpha$ phosphorylation and that the phosphorylation of PTP $\alpha$ may be a consequence of, rather than a prerequisite for, FAK-Src-PTK activation $(16,18)$. In mouse embryonic fibroblasts, it has been observed that PTP $\alpha$ was phosphorylated in response to integrin stimulation and required SFKs (Src or Fyn/Yes), FAK and an intact cytoskeleton. This suggested that the phosphorylation of PTP $\alpha$-Tyr789 is mediated by the active SFK/FAK complex $(18,19)$. The present study confirmed this hypothesis and, to the best of our knowledge, was the first to demonstrate the phosphorylation of PTP $\alpha$-Tyr789 by an upstream activator, leading to Src activation. The majority ( $20 \%)$ of Tyr789 phosphorylation is bound to Grb2 in NIH3T3 cells (19), however, this was not observed in $>20 \%$ of the endogenous PTP $\alpha$-Tyr789 in the present study, suggesting that free PTP $\alpha$-Tyr789 displaces the pTyr530 binding to the Src SH2 domain for Tyr530 dephosphorylation. However, other studies have demonstrated that the dephosphorylation of pTyr530 also occurs in the Tyr789-independent activation of Src under certain circumstances and in other cell types $(18,20)$. Therefore, the role of tyrosine phosphorylation of PTP $\alpha$ in Src activation remains to be elucidated.

Previous studies have demonstrated that PTP $\alpha$ dephosphorylates Tyr419 and Tyr530 at $\operatorname{Src}(8,11,21,22)$, leading to $\mathrm{Src}$ kinase activation. This indicates that Tyr789 leads to substrate specificity, not overall catalytic activity and is important in overexpressing the FAK-Del33 protein in breast cancer cells.

In conclusion, the present study revealed an unanticipated role for the FAK-Del33 protein in the regulation of PTP $\alpha$ phosphorylation, which is important in Src-Tyr530 dephosphorylation and, consequently, in enhancing cell migration and invasion.

\section{Acknowledgements}

This project was funded by Shanghai Committee of Science and Technology, China (grant no. 15ZR1426800). It was also funded by the National Natural Science Foundation of China under the grant 81071745 .

\section{References}

1. Stoker AW: Protein tyrosine phosphatases and signaling. J Endocrinol 185: 19-33, 2005.
2. Parsons JT: Focal adhesion kinase: the first ten years. J Cell Sci 116: 1409-1416, 2003.

3. Frame MC, Patel H, Serrels B, et al: The FERM domain: organizing the structure and function of FAK. Nat Rev Mol Cell Biol 11: 802-814, 2010.

4. Zhao X and Guan JL: Focal adhesion kinase and its signaling pathways in cell migration and angiogenesis. Adv Drug Deliv Rev 63: 610-615, 2011.

5. Klinghoffer RA, Sachsenmaier C, Cooper JA, et al: Src family kinases are required for integrin but not PDGFR signal transduction. EMBO J 18: 2459-2471, 1999.

6. Salazar EP and Rozengurt E: Src family kinases are required for integrin-mediated but not for $\mathrm{G}$ protein-coupled receptor stimulation of focal adhesion kinase autophosphorylation at Tyr-397. J Biol Chem 276: 17788-17795, 2001.

7. Lim ST, Mikolon D, Stupack DG, et al: FERM control of FAK functions: implications for cancer therapy. Cell Cycle 7: 2306-2314, 2008.

8. Zheng XM, Wang Y and Pallen CJ: Cell transformation and activation of pp60c-src by overexpression of a protein tyrosine phosphatase. Nature 359: 336-339, 1992.

9. Ponniah S, Wang DZ, Lim KL, et al: Targeted disruption of the tyrosine phosphatase PTPalpha leads to constitutive downregulation of the kinases Src and Fyn. Curr Biol 9: 535-538, 1999.

10. Arias-Salgado EG, Haj F, Dubois C, et al: PTP-1B is an essential positive regulator of platelet integrin signaling. J Cell Biol 170: 837-845, 2005.

11. Zheng XM, Resnick RJ and Shalloway D: A phosphotyrosine displacement mechanism for activation of Src by PTPalpha. EMBO J 19: 964-978, 2000.

12. Vacaru AM and den Hertog J: Serine dephosphorylation of receptor protein tyrosine phosphatase alpha in mitosis induces Src binding and activation. Mol Cell Biol 30: 2850-2861, 2010.

13. Fang XQ, Liu XF and Yao L: Somatic mutational analysis of FAK in breast cancer: a novel gain-of-function mutation due to deletion of exon 33. Biochem Biophys Res Commun 443: 363-369, 2014.

14. Zheng XM, Resnick RJ and Shalloway D: Mitotic activation of protein-tyrosine phosphatase alpha and regulation of its Src-mediated transforming activity by its sites of protein kinase C phosphorylation. J Biol Chem 277: 21922-21929, 2002.

15. Schlaepfer DD and Hunter T: Evidence for in vivo phosphorylation of the Grb2 SH2-domain binding site on focal adhesion kinase by Src-family protein-tyrosine kinases. Mol Cell Biol 16: 5623-5633, 1996.

16. Zeng L, Si X, Yu WP, et al: PTP alpha regulates integrin-stimulated FAK autophosphorylation and cytoskeletal rearrangement in cell spreading and migration. J Cell Biol 160: 137-146, 2003

17. Harder KW, Moller NP, Peacock JW, et al: Protein-tyrosine phosphatase alpha regulates Src family kinases and alters cell-substratum adhesion. J Biol Chem 273: 31890-31900, 1998.

18. Chen M, Chen SC and Pallen CJ, Integrin-induced tyrosine phosphorylation of protein-tyrosine phosphatase-alpha is required for cytoskeletal reorganization and cell migration. J Biol Chem 281: 11972-11980, 2006.

19. den Hertog J, Tracy S and Hunter T: Phosphorylation of receptor protein-tyrosine phosphatase alpha on Tyr789, a binding site for the $\mathrm{SH} 3-\mathrm{SH} 2-\mathrm{SH} 3$ adaptor protein GRB-2 in vivo. EMBO J 13: 3020-3032, 1994.

20. Yang LT, Alexandropoulos K and Sap J: c-SRC mediates neurite outgrowth through recruitment of Crk to the scaffolding protein Sin/Efs without altering the kinetics of ERK activation. J Biol Chem 277: 17406-17414, 2002.

21. Huang J, Yao L, Xu R, et al: Activation of Src and transformation by an RPTPalpha splice mutant found in human tumours. EMBO J 30: 3200-3211, 2011.

22. Wang J, Yu L and Zheng X: PTPalpha-mediated Src activation by EGF in human breast cancer cells. Acta Biochim Biophys Sin (Shanghai) 45: 320-329, 2013. 